\title{
The long way toward understanding host and viral determinants of therapeutic success in $\mathrm{HCV}$ infection
}

\author{
Pierre-Alain Rubbo $\cdot$ Philippe Van de Perre \\ Edouard Tuaillon
}

Received: 16 September 2011 / Accepted: 27 December 2011/Published online: 14 January 2012

(C) Asian Pacific Association for the Study of the Liver 2012

The T helper type 1 (Th1)/Th2 hypothesis of immune regulation arose in the late 1980 s, stemming from observations in mice [1]. Since then, the Th1/Th2 cytokine balance has been extensively described in humans as a pivotal component of antiviral immunity and pathogenesis in infections. In a simplistic view of the literature, Th1 cells are commonly associated with interferon-gamma (IFN- $\gamma$ ), interleukin (IL)-2 and IL-12 secretion, and these cells are hypothesized to mediate the response against intracellular pathogens such as viruses. Inversely, Th2 cells, which are most heavily reliant on IL-4, IL-5, IL-10 and IL-13, are supposed to emphasize protection against extracellular pathogens. In its modern incarnation, the role played by the Th1/Th2 balance in the regulation of cellular immunity against infections seems vaster and influenced by a large number of natural variables [2, 3]. In addition, since 1986 and the emergence of the Th1/Th2 hypothesis, other T-cell subpopulations, such as Th17 cells, have been described as functionally integrated actors of the antiinfectious immunity and crucial component of an effective response against pathogens [4]. Despite increasing evidence demonstrating the importance of these subpopulations in the effectiveness of the immune response against pathogens, the $\mathrm{Th} 1 / \mathrm{Th} 2$ balance remains a critical ratio to clear invaders.

For hepatitis $\mathrm{C}$ virus (HCV), a robust Th1 cell response and a mild Th2 activity, with a vigorous multi-epitopespecific $\mathrm{CD}^{+}{ }^{+}$and $\mathrm{CD}^{+}$T-cell responses, are associated with HCV clearance in chronic infection [5]. IFN- $\gamma$, a cytokine strongly associated with the Th1 response, exerts

P.-A. Rubbo $(\varangle) \cdot$ P. Van de Perre · E. Tuaillon INSERM U1058, CHU Montpellier and Montpellier 1 University, Montpellier, France

e-mail: pierrealainrubbo@gmail.com direct and durable antiviral effects inhibiting the replication of subgenomic and genomic HCV RNAs by stimulating the cytotoxic T-lymphocyte response. In patients with chronic hepatitis $\mathrm{C}$, the cytotoxic $\mathrm{CD} 8^{+} \mathrm{T}$-cell activity may also be inversely related to the viral load.

Viral sequence mutations are an important mechanism to escape the adaptative cellular immune response. A high level of replication, high mutation rate (estimated to be 1 in 1000 bases per year) due to the lack of proof-reading capacity of its polymerase, and pre-existing quasi-species contribute to the rapid diversification of the viral population and limits the efficiency of an adaptive immune response [6].

In addition, infiltrated cells in the liver belong mostly to the Th1 cells, unable to secrete IL-4 and IL-5 [7]. In this context, the overproduction of the initially protective immune response and soluble factors at the site of infection leads to adverse effects resulting in hepatocellular damage. Th1 cells release TNF- $\alpha$, IFN- $\gamma$, and IL- 2 causing selfinflicted inflammation and necrosis. To limit these events and the persistence of $\mathrm{HCV}$, the Th1 immune response must be kept under control. Th2 cells such as IL-4- and IL-10-secreting cells have a vital role in protecting the host from the potentially damaging consequences of Th1 cells in chronic HCV infection by suppressing the Th1 response and counteracting the fibrogenic effects of TNF- $\alpha$, IFN $-\gamma$, and IL-2. On the other hand, the Th2 response is also more frequently associated with viral persistence and resistance to treatment in contrast to the viral clearance associated with Th1 [8]. Hence, intrahepatic IL-10-producing T cells are more frequently detected in patients with chronic hepatitis. An increased Th1 and Th2 response has been shown during chronic $\mathrm{HCV}$ infection with a greater elevated level of Th2 than Th1 cytokines [9]. This suggests that an enhanced Th2 response is at least partly responsible 
for immunopathogenesis of chronic $\mathrm{HCV}$ infection and allows for the suppression of the immune response, contributing to the inability to eliminate the virus.

It is also known that the innate immune response and especially NK cells play a crucial role in controlling $\mathrm{HCV}$ infections through the recognition of virally infected cells by inhibitory NK cell receptors (KIR). In particular, genes encoding the KIR2DL3 directly influence antiviral immunity and protection against $\mathrm{HCV}$ by providing a weaker inhibitory signal, which results in weaker inhibition of NK cells [10]. Nevertheless, these data were only reported from Caucasian and African American subjects and, to our knowledge, never from Asian people.

The standard treatment of patients infected with chronic $\mathrm{HCV}$ infection is a combination therapy with pegylated interferon $\alpha-2 b$ and ribavirin [11]. This combination is not only associated with antiviral effects and sustained virological response, but also with pleiotropic immunomodulatory and anti-fibrotic properties that cause significant changes in the Th1/Th2 balance [5]. Ribavirin is also associated with the mutation of HCV RNA and activation of host cellular immunity [12]. To date, the response to IFN-based therapy remains poorly understood, but several host and virus markers associated with recovery have been identified. Under treatment, the predictive response varies according to $\mathrm{HCV}$ genotypes. In general, genotype 1 is more resistant to the combination of therapy than genotypes 2 and 3 that are predictors of a sustained response. IL-28B polymorphism and the IFN- $\gamma$-induced protein 10 (also named CXCL-10) baseline concentrations are associated with the therapeutic response. Several other factors, such as HCV RNA levels, the stage of the liver disease and infection, the patient's age, the viral genotype, sex, race, body mass index, and host immunological parameters are also suggested to play a role in determining the therapeutic response [13]. Nevertheless, the impact of IFN-based therapy on host virus interplay could be viewed as a black box, since the determinants of response to treatment remain largely unknown. Serum levels of MIG and IP-10 in sustained virological responders decreased after the initiation of therapy, whereas the level of RANTES remained identical throughout the treatment [8]. The pattern of the chemokine expression levels may therefore indicate that the original levels of MIG and IP-10 are related to the rate of viral clearance. Treatment is also accompanied by a decreased secretion of the Th2 cytokines such as IL-4 and IL-10. A lower Th1/Th2 ratio before IFN monotherapy was a significant host factor for predicting long-term virological response in chronic $\mathrm{HCV}$ infection [13]. Further investigations of immunological mechanisms and HCV factors associated with successful therapeutic response are needed to get away from a blind immunotherapy.
The viral nonstructural 5A (NS5A)2209-2248 genomic region has been characterized as an interferon sensitivitydetermining region (ISDR) and was suggested as a predictor of the treatment response. In particular, the histidine residue at position 2218 seemed to have a critical effect on IFN sensitivity. NS5A harbors T-cell epitope and is not directly affected by neutralization [14]. A higher number of amino acid substitutions in the ISDR was strongly associated with a favorable response to IFN therapy in patients infected with genotype $1 \mathrm{HCV}$ [15] and, according to Ishii et al. in this journal [16], with a higher proportion of Th2 cells. Although the exact role of NS5A remains to be elucidated, this protein has been shown to bind a range of cellular signaling molecules that may affect numerous cellular pathways in $\mathrm{HCV}$-infected cells, including the antiviral IFN $-\alpha$ response. The antiviral effect of these mutations within NS5A may be associated with the disruption of the interaction between NS5A and RNAdependent protein kinase (PKR) that renders $\mathrm{HCV}$ sensitive to treatment.

These mutations are the consequences of HCV intrahost evolution, which is characterized by perpetual changes in the genome for escaping from the host-adaptive antiviral response. Amino acid exchanges observed in the ISDR are mostly due to natural variability of $\mathrm{HCV}$ without specific selective pressure due to treatment [17]. In Japan, mutations in the ISDR gene were strongly associated with the sustained virological response to IFN therapy in patients infected with genotype $1 \mathrm{~b}$, while the likelihood of this response is less pronounced in Europe suggesting geographical differences in the relationship between ISDR mutations and efficiency of IFN therapy. In a recent study, dynamics of intrahost HCV variants of the NS5A genomic region were sequenced from four treatment-naïve chronically infected patients followed up for 9-18 years from the acute infection stage [18]. The authors established a model of $\mathrm{HCV}$ evolution in four stages. In stage one, $\mathrm{HCV}$ seeding of a new host with $\mathrm{HCV}$ variants representing different viral communities upon transmission ensures the establishment of the extensive sequence diversity and broad community structure of the $\mathrm{HCV}$ population in the context of adaptative immune responses. In stage two, there is an incremental evolution of viral variants within predominant communities. Stage three characterizes a period of diversification into a set of subpopulations that become prominent with the decline of the previously dominant population. Stage four corresponds to a period of a strong negative selection, which allows HCV to achieve a stable adaptation to the host, the ultimate goal of intrahost evolution. The authors added that HCV may be differentially sensitive to interventions at particular stages, which should help in determining the optimal timing for therapy. For example, patients with acute infection (stages one and 
two) reach a complete virological response to treatment more frequently than patients with chronic infections. Also, variation in quasi-species diversity throughout the course of $\mathrm{HCV}$ infection may be related to the reduction in the selection intensity, which is the result of a decline in specific immune responses even if $\mathrm{HCV}$ does not cause systemic immunodeficiency [18]. Therefore, it is conceivable that a viral population at a given stage has a specific impact on host immunological properties suggesting that the relationships between HCV variants, immune parameters, and response to IFN-based treatment are highly complex. Although these observations may indicate an association between the timing, pattern, and specificity of the cellular immune response, and emergence of ISDR mutations on NS5A in predominant HCV quasi-species, they do not establish whether ISDR mutations directly affect IFN functions or are only a marker of a specific host-virus interaction in which providing IFN is especially useful to $\mathrm{HCV}$ recovery. The Th1/Th2 balance is a crucial component of the treatment response against $\mathrm{HCV}$, but is probably not directly linked to ISDR mutations. The effective host response under treatment is likely a consequence of a more complex integrated network of immunological factors involved in this antiviral response under treatment. Therefore, more evidence is needed concerning the role played by ISDR mutations in the Th1/Th2 ratio associated with an effective antiviral response under treatment.

In the current issue of Hepatology International, Ishii et al. [16] investigated the impact of amino acid mutations in the ISDR of the NS5A HCV-1b protein on the host Th1/Th2 subpopulations throughout the course of standard therapy. In this retrospective study, the authors first compared clinical data of two homogeneous groups of $\mathrm{HCV}$ $1 b$-infected patients defined by the number of mutations in their viral ISDR sequence. They then observed the kinetics of viral replication and host immunity by evaluating the proportion of $\mathrm{Th} 1 / \mathrm{Th} 2 \mathrm{CD}^{+}{ }^{+} \mathrm{T}$-cell populations, when therapeutic ribavirin combined with either IFN- $\alpha 2 b$ or pegylated IFN- $\alpha 2 b$ was given to the HCV-1b-infected patients. In the first part, the authors selected 44 patients with chronic hepatitis C between 2002 and 2006. Enrollment criteria comprised age, high HCV RNA levels before treatment, and infection with HCV genotype 1b. Once stratified according to the number of mutations detected in viral ISDR, no statistical differences in clinical characteristics were observed between the two groups of patients at the baseline except that the group with more mutations had more frequently males ( 83 vs. $38 \%$ ). Similarly, they found that patients belonging to the group with a higher number of ISDR mutations had higher concentrations of hemoglobin and ferritin. In the second part, the viral kinetics were analyzed by estimating the rate of patients having a rapid or early viral responses under treatment, defined as patients with a serum HCV-1b RNA level $<50 \mathrm{IU} / \mathrm{ml}$ at 4 or 12 weeks after starting therapy. Patients with more mutations in the ISDR of viral NS5A cleared the virus faster than patients with fewer mutations. Indeed, the authors report that $30 \%$ of patients with more mutations can be defined as rapid responders, while $70 \%$ are early responders after treatment initiation. By comparison, they enumerated no rapid responders and only $14 \%$ of early responders in the group of patients displaying less mutations. Finally, the kinetics of host immunity were evaluated by comparing the proportion of $\mathrm{CD} 4^{+} \mathrm{T}$ cells producing IFN- $\gamma$ (defined as Th1 cells) and/or IL-4 (defined as Th2 cells) using flow cytometry before and at days 3, 7, 14, 28, and 56 after treatment initiation. The authors argue that they could not find any difference in the proportion of $\mathrm{CD}^{+}{ }^{+}$Th1 cells before and throughout the course of treatment between the two groups. In contrast, they observed that more mutations in ISDR were associated with a higher proportion of $\mathrm{CD}^{+}{ }^{+} \mathrm{Th} 2$ cells than fewer mutations before and at least at days 3, 7, 14, and 28 after treatment administration. This shows that IFN-based therapy has no effect on the proportion of Th2 cells between the two groups, since these cells had consistently higher proportion before and after treatment initiation than in patients with less than two mutations in the ISDR.

To our knowledge, this study is one of the first suggesting that a higher number of mutations in the ISDR and faster effective immune response may be associated with a higher prevalence of Th2 cells in peripheral blood of HCV$1 b$-infected patients. The results reported here are therefore noteworthy in the context of host protection against $\mathrm{HCV}$, host/virus synergy, and further development of therapeutics. These data are concordant with other reports indicating that (1) a lower $\mathrm{Th} 1 / \mathrm{Th} 2$ ratio before treatment predicts a long-term virological response in patients with chronic HCV infection [13], and (2) the therapy seems to be more effective in $\mathrm{HCV}$-infected patients with a predominant $\mathrm{Th} 2$ profile or with low levels of IP-10 [19]. Nevertheless, some points need substantial clarification and several conclusions need to be taken with caution.

First, although several baseline parameters, such as age, HCV genotype, IFN-based therapy, length of treatment, viral load, and many biological factors were matched between the two groups, other important parameters potentially influencing the immunological environment or viral dynamics were lacking or were not discussed, although significantly different between the two populations of patients. In particular, there are more males than females in the group having more than two mutations in the ISDR of HCV NS5A. This is important because some reports show that sex could be associated with a sustained virological response, although this conclusion is still subject to controversy [20]. It has been described that sex may 
impact Th1/Th2 cytokine production in response to certain infections and treatment. Also, ethnicity and body mass index have not been taken into account in this study, although they have been previously shown as important parameters [13], playing a role in determining the therapeutic efficacy. Despite the difference being not significant in the present study, total cholesterol levels between the two groups of patients have not been taken into account. Similarly, the higher baseline levels of hemoglobin and ferritin in the group with two or more ISDR mutations are not discussed, although both these parameters have been previously associated with an improved therapeutic response or therapeutic failure, respectively.

Second, mutations found in the NS5A ISDR sequences have not been given and the relative distribution of each variant among $\mathrm{HCV}$-infected patients. As said previously, these mutations in the ISDR of the viral NS5A may be associated with the disruption of the interaction between NS5A and PKR that may restore treatment efficiency against $\mathrm{HCV}$. An in-depth analysis of $\mathrm{HCV}$ variants could have brought some clues on the timing of infection and, thus, its role in the immunomodulation of the Th1/Th2 ratio. Also, monitoring ISDR mutations throughout the therapy could help explain the dissimilar profile of peripheral blood Th2 cells in the two groups of patients. This could be important to confirm that IFN-based treatment and/or HCV variants have direct effects on immune regulation and $\mathrm{Th} 1 / \mathrm{Th} 2$ ratio.

Third, since polyfunctionality of $\mathrm{T}$ cells is essential for an effective protective immune response and clearing the pathogens, concomitant investigations of several other cytokines and chemokines involved in the Th1, Th2, and also Th17 pathways would probably reinforce and make more complex the hypothesis developed by Ishii et al. In this regard, the measurement of cytokine/chemokine secretions in T-cell culture supernatants by multiplex microbead assays can be useful [2, 3].

Fourth, as Ishii et al. enrolled patients selected in their hospital in Tokyo, Japan, we can presume that the majority of them, if not all, are Japanese people. It is important because some reports showed that ISDR mutations are more important in Japanese patients infected with HCV-1b isolates with different biological properties than, for example, in Caucasian patients. Therefore, we can assume that these results may unlikely be similar when investigated in other populations.

Finally, altogether these data suggest that ISDR mutations may not directly favor a better immune response against $\mathrm{HCV}$, also considering patients under therapy, but can also be a marker of immunological status and virus evolution. The real biological advantage of individuals belonging to the ISDR $\geq 2$ group may be the lower $\mathrm{Th} 1 / \mathrm{Th} 2$ ratio at the baseline compared to patients with less than two mutations. In the latter case, this ratio may be viewed as unbalanced in favor of the Th1 response. Under the administration of Th1-type IFN-based therapy, HCVinfected patients with a lower natural Th1 response, corresponding to the ISDR $\geq 2$ group, would be therefore more responsive to therapy, which is more effective compared to the other group. In such circumstances, this work emphasizes the complex relationships between $\mathrm{HCV}$, host immune response and therapy, and has the virtue of proposing an immunological view of the benefit of mutations in ISDR during the therapeutic response. Nevertheless, many efforts need to be made before we can fully understand the mechanisms making the ISDR mutations a benefit for the immune response and treatment outcome.

\section{References}

1. Mosmann TR, Cherwinski H, Bond MW, Giedlin MA, Coffman RL. Two types of murine helper $\mathrm{T}$ cell clone. I. Definition according to profiles of lymphokine activities and secreted proteins. J Immunol 1986;136:2348-2357

2. Rubbo PA, Tuaillon E, Bollore K, et al. The potential impact of $\mathrm{CD}^{+}{ }^{+} \mathrm{T}$ cell activation and enhanced $\mathrm{Th} 1 / \mathrm{Th} 2$ cytokine ratio on HIV-1 secretion in the lungs of individuals with advanced AIDS and active pulmonary infection. Clin Immunol 2011;139:142-154

3. Rubbo PA, Tuaillon E, Nagot N, et al. HIV-1 infection impairs HSV-specific $\mathrm{CD}^{+}$and $\mathrm{CD} 8^{+} \mathrm{T}$-cell response by reducing Th1 cytokines and CCR5 ligand secretion. J Acquir Immune Defic Syndr 2011;58:9-17

4. Dubin PJ, Kolls JK. Th17 cytokines and mucosal immunity. Immunol Rev 2008;226:160-171

5. Cramp ME, Rossol S, Chokshi S, Carucci P, Williams R, Naoumov NV. Hepatitis C virus-specific T-cell reactivity during interferon and ribavirin treatment in chronic hepatitis C. Gastroenterology 2000;118:346-355

6. Farci P, Shimoda A, Coiana A, et al. The outcome of acute hepatitis $\mathrm{C}$ predicted by the evolution of the viral quasi species. Science 2000;288:339-344

7. Bertoletti A, D'Elios MM, Boni C, et al. Different cytokine profiles of intraphepatic $\mathrm{T}$ cells in chronic hepatitis B and hepatitis C virus infections. Gastroenterology 1997;112:193-199

8. Wan L, Kung YJ, Lin YJ, et al. Th1 and Th2 cytokines are elevated in $\mathrm{HCV}$-infected SVR(-) patients treated with interferon-alpha. Biochem Biophys Res Commun 2009;379:855-860

9. Fan XG, Liu WE, Li CZ, et al. Circulating Th1 and Th2 cytokines in patients with hepatitis $\mathrm{C}$ virus infection. Mediators Inflamm 1998;7:295-297

10. Khakoo SI, Thio CL, Martin MP, et al. HLA and NK cell inhibitory receptor genes in resolving hepatitis $\mathrm{C}$ virus infection. Science 2004;305:872-874

11. Fried MW, Shiffman ML, Reddy KR, et al. Peginterferon alfa-2a plus ribavirin for chronic hepatitis $\mathrm{C}$ virus infection. N Engl $\mathrm{J}$ Med 2002;347:975-982

12. Lau JY, Tam RC, Liang TJ, Hong Z. Mechanism of action of ribavirin in the combination treatment of chronic $\mathrm{HCV}$ infection. Hepatology 2002;35:1002-1009

13. Shirakawa H, Matsumoto A, Joshita S, et al. Pretreatment prediction of virological response to peginterferon plus ribavirin therapy in chronic hepatitis $\mathrm{C}$ patients using viral and host factors. Hepatology 2008;48:1753-1760 
14. Macdonald A, Harris M. Hepatitis C virus NS5A: tales of a promiscuous protein. J Gen Virol 2004;85:2485-2502

15. Enomoto N, Sakuma I, Asahina Y, et al. Mutations in the nonstructural protein $5 \mathrm{~A}$ gene and response to interferon in patients with chronic hepatitis $\mathrm{C}$ virus $1 \mathrm{~b}$ infection. $\mathrm{N}$ Engl $\mathrm{J}$ Med 1996;334:77-81

16. Ishii K, Shinohara M, Kogame $M$ et al. Effects of mutation number in interferon sensitivity determining region on peripheral blood $\mathrm{CD}^{+}{ }^{+} \mathrm{T}$ cell subsets (Th1, Th2) in chronic hepatitis $\mathrm{C}$ patients with hepatitis $\mathrm{C}$ virus genotype $1 \mathrm{~b}$ and high viral load. Hepatol Int. 2011. doi:10.1007/s12072-011-9305-8

17. Rispeter K, Lu M, Zibert A, Wiese M, de Oliveira JM, Roggendorf $\mathrm{M}$. The "interferon sensitivity determining region" of hepatitis C virus is a stable sequence element. J Hepatol 1998;29: $352-361$
18. Ramachandran S, Campo DS, Dimitrova ZE, Xia GL, Purdy MA, Khudyakov YE. Temporal variations in the hepatitis $\mathrm{C}$ virus intrahost population during chronic infection. J Virol 2011;85: 6369-6380

19. Lagging M, Romero AI, Westin J, et al. IP-10 predicts viral response and therapeutic outcome in difficult-to-treat patients with HCV genotype 1 infection. Hepatology 2006;44:1617-1625

20. Akuta N, Suzuki F, Kawamura Y, et al. Predictive factors of early and sustained responses to peginterferon plus ribavirin combination therapy in Japanese patients infected with hepatitis $\mathrm{C}$ virus genotype $1 \mathrm{~b}$ : amino acid substitutions in the core region and lowdensity lipoprotein cholesterol levels. J Hepatol 2007;46:403-410 